\title{
Recent Developments in Enantioselective Phase Transfer Catalysis Using Chiral Ammonium Salts
}

\author{
Jérôme Vachon and Jérôme Lacour*
}

\begin{abstract}
Due to the constant demand for non-racemic molecules in both industrial and academic laboratories, many efforts have been devoted in the last 25 years to the development of enantioselective phase transfer catalyzed (PTC) processes. Much of this chemistry was recently reviewed - the latest reports were from 2003/2004 and treated essentially the synthesis of non racemic $\alpha$-amino acids. After a short historical introduction, this article will thus review enantioselective PTC reactions reported in the last three years (2003-2006) and demonstrate how broad and active the field is - and this considering ammonium-catalyzed reactions only.
\end{abstract}

Keywords: Ammonium salts · Catalysis · Chirality · Green chemistry · Phase transfer

Since the pioneering work of Makosza and coworkers almost forty years ago [1][2], and the extensive study of Starks et al. in the early 1970s [3][4], phase transfer catalysis (PTC) has become a topic of great interest belonging, nowadays, to the burgeoning field of organocatalysis [5]. PTC involves setting up experimental conditions that allow reactions to proceed through the active transport of reactive polar reagents from one liquid phase to another by small sub-stoichiometric amounts of (charged) molecules. It has many advantages over other catalytic (and stoichiometric) processes: simple reaction procedure, mild conditions, safe, inexpensive and environmentally friendly reagents, absence of anhydrous or aprotic solvents, ease of scaleup and (most often) metal-free conditions. Moreover, PTC is compatible with a wide range of solvents (if immiscible with water which usually contains the reactive polar reagents), with ionic liquids [6], and can even be carried out without any organic solvent if the substrate plays the role of the organic phase itself [7][8]. All in all, PTC can be considered a 'green' alternative to many classical homogeneous reaction conditions [9].

Due to the constant demand for nonracemic molecules in both industrial and academic laboratories, many efforts have been devoted in the last 25 years to the development of enantioselective processes using enantiopure (organic) moieties as chiral phase transfer catalysts. Much of this chemistry was recently reviewed - the latest reports were from 2003 and 2004 and treated essentially the synthesis of nonracemic $\alpha$-amino acids [10-12]. After a short historical introduction, this article will thus describe enantioselective PTC reactions reported in the last three years (2003-2006) and demonstrate how broad and active the field is ( 90 references) - and this considering ammonium catalyzed reactions only.

\section{Milestones of Enantioselective PTC Before 2003}

In 1984, chemists at Merck were the first to report an efficient enantioselective PTC reaction using an $\mathrm{N}$-benzylcinchoninium bromide salt as catalyst for the methylation of a phenyl-indanone substrate (Scheme 1); the base-induced reaction proceeded with 95\% yield and $92 \%$ enantiomeric excess (ee) [13].

In 1989, O'Donnell and co-workers showed that non-racemic natural and unnatural $\alpha$-amino acids could be readily synthesized using the Schiff base of $t$-butyl glycinate 1, stoichiometric amounts of inorganic aqueous bases and alkyl halides as electrophiles and, again, cinchona ammonium derivatives as phase-transfer catalysts; the enantiomeric excess of the alkylated products varying from 42 to $66 \%$ (Scheme 2) [14].

Since then and for many years, alkylation reactions of ester $\mathbf{1}$ have been the most

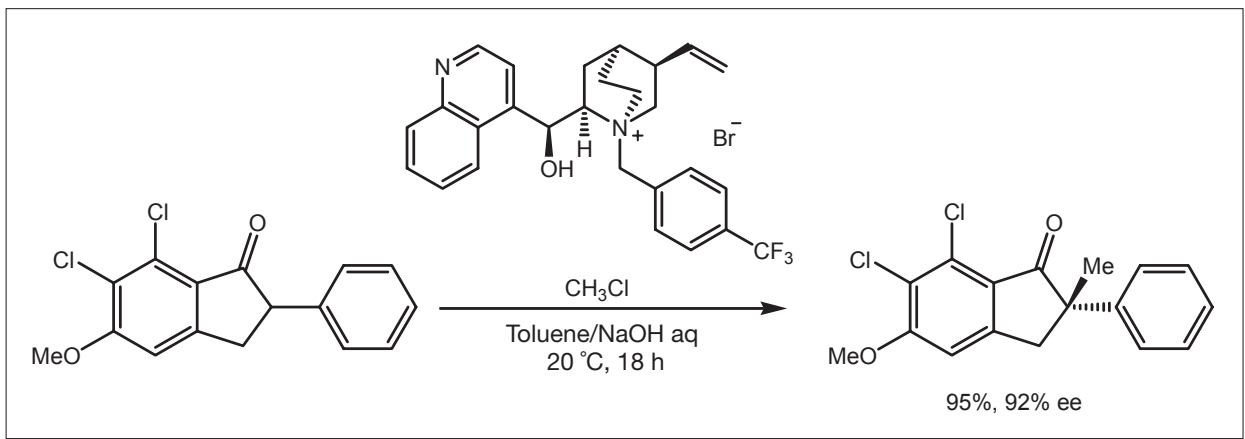

Scheme 1 


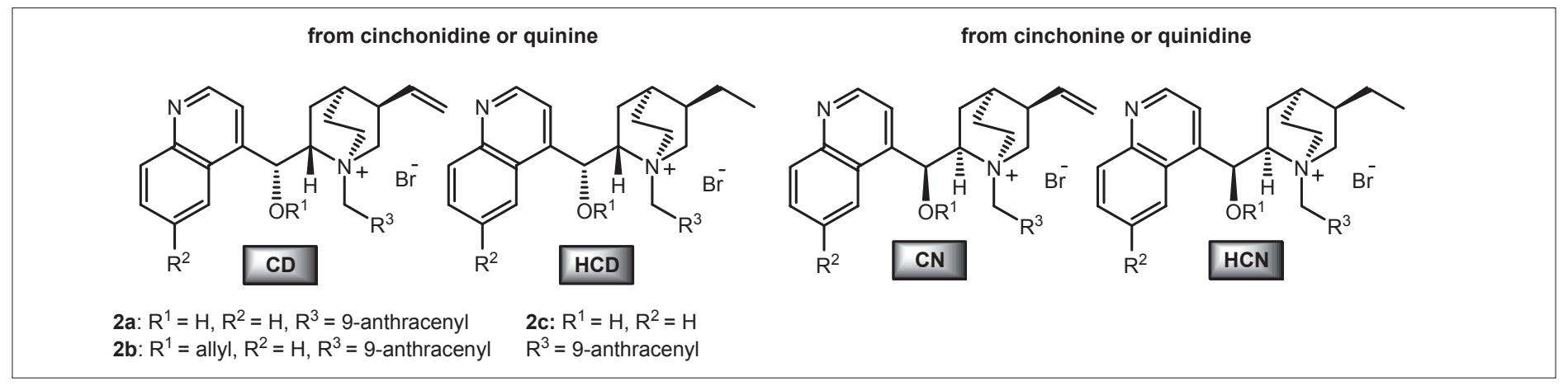

Fig. 1.

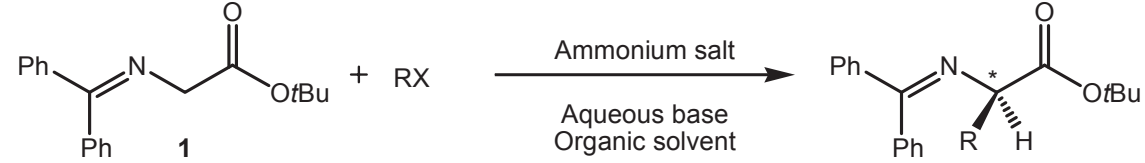

Scheme 2 .

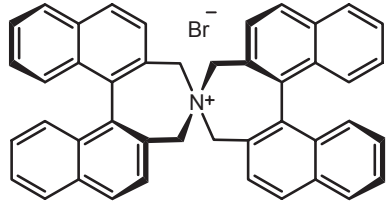

$(S, S)-3 a$

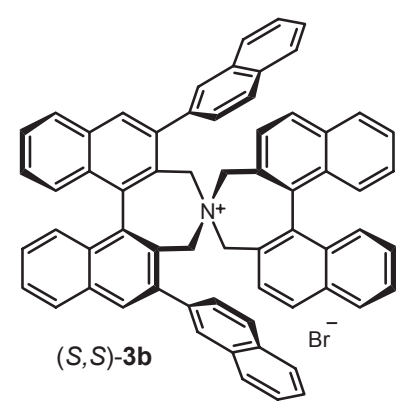

$(S, S)-\mathbf{3 b}$ results being obtained with salts of cation $3 \mathbf{b}$ that contains $\beta$-naphthyl substituents at the 3,3' positions of the binaphthyl core (ee $=96 \%$ ) [25].

After this intentionally short and limited summary of the situation up to 2003, care will now be taken to detail novel catalytic ammonium salts - without excluding the 'old faithfuls' that still find new and effective uses. To give a quick perception of the efficiency of the catalytic moieties, the best enantiomeric excesses obtained with them will be indicated close to the structures with a few words describing the chemical context of their use - the reactions being detailed later in the review according to the substrate nature, reagents and conditions.

\section{Non-racemic Ammonium Cations for Enantioselective PTC}

Chiral ammonium phase transfer catalysts can be divided in three main classes that are

(i) molecules derived from cinchona alkaloids (Fig. 3 and Fig. 4),

(ii) those made from other chiral pool moieties (Fig. 5) [26], and

(iii) purely synthetic ammonium cations (Fig. 6 and Fig. 7).

A fourth class will be briefly presented which is the group of the readily recyclable (including polymer supported) catalysts.

ported cinchona-alkaloid derivatives were also synthesized using Merrifield resins [22][23]; O-linked Merrifield polymers performing equally well [24].

However, fine tuning of cinchona-alkaloid ammonium catalysts is somewhat limited to the above described structural modifications and, in this context, quite a few groups have entered the field of enantioselective PTC by proposing novel structures and geometries for the ammonium moieties. For instance, in 1999, Maruoka and coworkers showed that purely synthetic $C_{2}$-symmetrical bis(binaphthyl)ammonium salts of type 3 (Fig. 2), available from $(S)$ or $(R)$-BINOL in several steps, are effective chiral phase transfer catalysts. The reactions of ester 1 with alkyl halides occurred with decent selectivity using the 'naked' ammonium cation 3a (up to $79 \%$ ee); better

\subsection{Cinchona-based Ammonium Cations}

As mentioned, several modifications of the cinchona alkaloid core have been proposed that involve, in general, introduction of substituents (i) at the bridgehead nitrogen atom, (ii) at the benzylic hydroxyl group or (iii) at position 6 of the quinoline. The efficiency of the catalysts relies mostly on the substituent added on the bridgehead nitrogen atom. Various benzylic groups have been introduced to study the influence of steric effects (e.g. compounds 4 and 5) [27][28], of electronic effects (molecule 6) [29], and a combination of both (7 and 8) [30][31]. Taking into account these factors, it is possible to elaborate the right catalyst were linked to the bridgehead nitrogen an to the benzylic oxygen atoms. Catalyst op- 


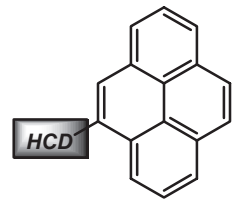

$$
\text { 4: } R^{1}=H, R^{2}=H \text {, ee up to } 94 \%
$$
(Schiff base ester alkylation)

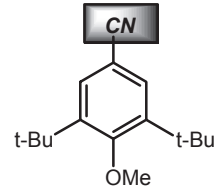

7a: $R^{1}=$ allyl, $R^{2}=H$, ee. up to $99 \%$ (alkylation of $\alpha, \beta$-keto esters)

$$
7 \mathbf{b}: \mathrm{R}^{1}=\text { propargyl, } \mathrm{R}^{2}=\mathrm{H}
$$

to $76 \%$ (fluorination of $\alpha$-cyano esters) ee up to $69 \%$ (fluorination of $\alpha, \beta$-keto esters)

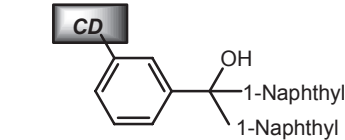

5: $R^{1}=$ allyl, $R^{2}=H$, ee up to $99 \%$ (Schiff base ester alkylation)

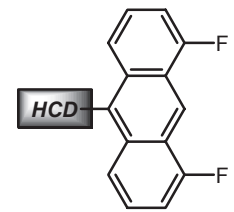
8: $\mathrm{R}^{1}=$ allyl, $\mathrm{R}^{2}=\mathrm{H}$, ee up to $98 \% 9: \mathrm{R}^{1}=\begin{aligned} & p \text {-Tosyl, } \mathrm{R}^{2}=\mathrm{H}, \text { ee up to } 95 \% \\ & \text { (aziridination of olefins) }\end{aligned}$
Fig. 3. Monomeric cinchona ammonium catalysts - structural variation of the $\mathrm{R}^{3}$ group in reference to structures detailed in Fig. 1

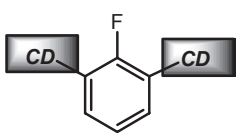

10a: $R^{1}=$ allyl, $R^{2}=H$, ee up to $99 \%$ (Schiff base ester alkylation)

10b: $R^{1}=H, R^{2}=$ OMe, ee up to $99 \% \quad 11: R^{1}=$ allyl, $R^{2}=H$, ee up to $99 \%$ (epoxidation of chalcone)

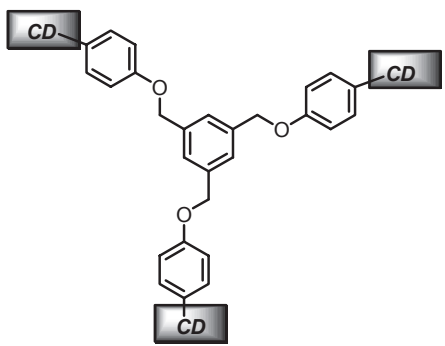

12: $R^{1}=$ allyl, $R^{2}=H$, ee up to $98 \%$ (Schiff base ester alkylation)
Fig. 4. Dimeric and trimeric cinchona ammonium catalysts - structural variation of the $\mathrm{R}^{3}$ group in reference to structures detailed in Fig. 1

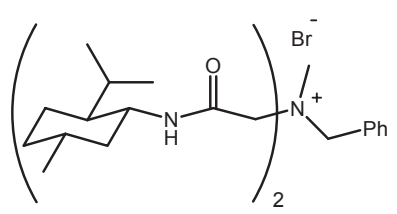

13: ee up to $72 \%$ (Schiff base ester alkylation)<smiles>CCCCC[C@@H](C)C1(c2ccccc2)O[C@H](C[N+]2(C)[C@H](C)C=C[C@H]2C)C=C[C@H]1C</smiles>

15b: ee up to $37 \%$ (Schiff base ester alkylation)

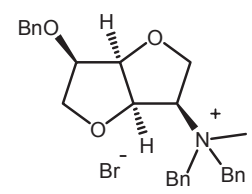

14: ee up to $48 \%$ (Schiff base ester alkylation)

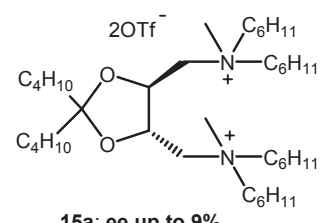

15a: ee up to $9 \%$ ee up to $11 \%$
(Michael addition on $\alpha, \beta$-unsaturated esters)

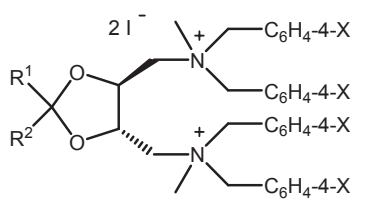

16a: $R^{1}=t B u, R^{2}=M e, X=O M e$ ee up to $94 \%$ (Schiff base ester alkylation)

16b: $R^{1}=R^{2}=i \operatorname{Pr}, X=M e$ ee up to $82 \%$

(Michael addition on $\alpha, \beta$-unsaturated esters)

16c: $\mathrm{R}^{1}=\mathrm{R}^{2}=\left(\mathrm{CH}_{2}\right)_{2}-\mathrm{C}_{6} \mathrm{H}_{4}-4-\mathrm{F}, \mathrm{X}=\mathrm{Me}$ de up to $90 \%$, ee up to $82 \%$ (Mannich reaction on Schiff base ester)

Fig. 5. for the desired reaction; compound 9 being for example designed for the aziridination of electron-deficient olefins [32].

As mentioned, effective dimeric and trimeric catalysts combining two or three cinchona ammonium cations were elaborated before 2003. Structural improvements were recently reported by extensive variations of the chemical nature of the linker (length, size, electronics) affording novel dimeric (10, 11) and trimeric (12) moieties; very high enantiomeric excesses being obtained in many of the studied reactions [33-36].

\subsection{Other Ammonium Cations Derived from Chiral Pool Moieties}

As one might suspect, the structural diversity of the chiral pool was exploited to generate non-racemic ammonium catalysts of structures different from those of the cinchona alkaloids. For instance, quaternary ammonium cations 13 and $\mathbf{1 4}$ (Fig. 5) were synthesized respectively from L-menthol and isosorbide, which is a byproduct of the starch industry from the dehydration of Dsorbitol [37][38].

Modest to decent levels of selectivity were obtained when these cations were reacted under PTC conditions. Ammonium cations derived from tartaric acid were also prepared (e.g. 15 and 16) and used successfully (vide infra). The nature of the substituents on the ammonium moieties is decisive in order to reach high level of enantioselectivity - this is particularly noticeable when comparing the efficiency of salts $\mathbf{1 5}$ and $\mathbf{1 6}$ [39-44].

\subsection{Purely Synthetic Ammonium Cations}

As mentioned, very high levels of enantioselectivity were obtained before 2003 when using spiro $C_{2}$-symmetrical bis(binaphthyl) ammonium salts of type $\mathbf{3} \mathbf{a}$ or $\mathbf{3 b}$. Further modifications of the nature of the aryl substituents at 3,3' positions (compounds $\mathbf{3 c}$ to 3f, Fig. 6) were studied by the Kyoto group [45][46]. The major drawback of molecules of type $\mathbf{3}$ is that more than ten steps are classically required to synthesize the appropriate binaphthyl subunits. In order to simplify the preparation of the compounds, Maruoka and coworkers developed a new synthetic procedure; one of the improvements being metallation of the binaphthyl core with magnesium bis(2,2,6,6-tetramethylpiperamide) as a base, which facilitates the scale-up of the overall process [47].

Introduction of aryl substituents at the $4,4^{\prime}$ and 6,6' positions of the bis-binaphthlyl core was also carried out (e.g. 17 and 18) [48][49]. Compound 18, which presents a $D_{2}$-symmetry, is interesting as its preparation requires essentially half the number of synthetic preparative steps compared to $C_{2}$ symmetrical analogues. Catalyst $\mathbf{1 9}$, based 


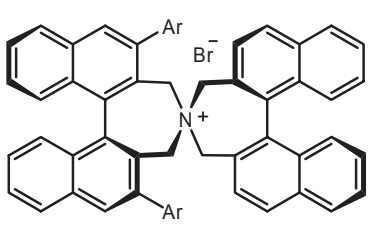

$(\mathrm{S}, \mathrm{S})-3 \mathrm{c}\left(\mathrm{Ar}=3,4,5-\mathrm{F}_{3}-\mathrm{Ph}\right)$

$(\mathrm{S}, \mathrm{S})-3 \mathbf{d}\left(\mathrm{Ar}=3,5\right.$-bisCF $\left.\mathrm{F}_{3}-\mathrm{Ph}\right)$

$(\mathrm{S}, \mathrm{S})$-3e $\left(\mathrm{Ar}=3,5\right.$-bis $\left.\left(3,5-\mathrm{bisCF}_{3}-\mathrm{Ph}\right)-\mathrm{Ph}\right)$

(S, S)-3f $(\mathrm{Ar}=3,5$-bis (3,5-bistBu-Ph)-Ph)

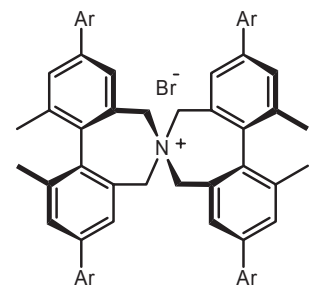

$(R, R)-\mathbf{1 9}\left(\mathrm{Ar}=\mathrm{C}\left(\mathrm{Me}_{2}\right) \mathrm{Ph}\right)$ ee up to $97 \%$ (Schiff base ester alkylation)

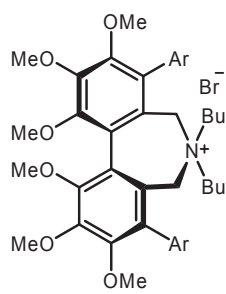

$(\mathrm{S}, \mathrm{S})-\mathbf{2 2}\left(\mathrm{Ar}=3,4,5-\mathrm{F}_{3}-\mathrm{Ph}\right)$ ee $=94-98 \%$ (Schiff base ester alkylation)

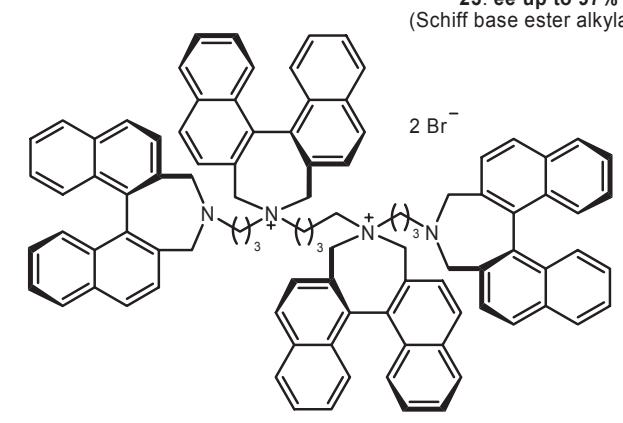

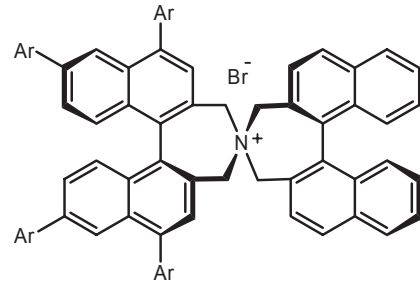

(S,S)-17 (Ar = 3,5-diphenylphenyl) (Schiff base ester alkylation)

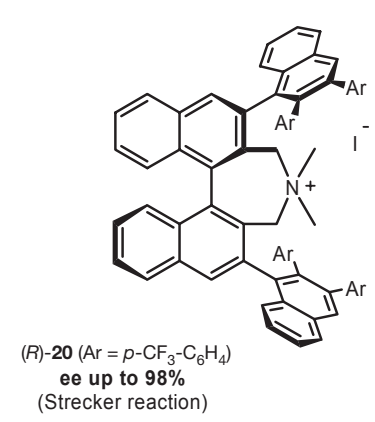<smiles>COc1cc(-c2cc(C(F)(F)F)cc(C(C)(C)C)c2OC)c2c(c1-c1cc(C(F)(F)F)cc(C(F)(F)F)c1)[N+](C)([C@H](c1cccc3ccccc13)C(F)(F)F)Cc1c-2cc(C(C)(C)C)c(OC)c1C(F)(F)F</smiles><smiles>C[C@@H]1CCC2=c3c4ccc(Br)cc(c(Br)cc3c4-c3cc(Br)cc4cc(Br)ccc34)C[NH+](C2)C1</smiles>

(S,S)-18 (Ar = 3,5-diphenylphenyl) (Schiff base ester alkylation)<smiles>Brc1cc2ccccc2c2c1C[N+](Br)(Br)Cc1c(Br)cc3ccccc3c1-2</smiles>

(S) $-21\left(\mathrm{Ar}=3,4,5-\mathrm{F}_{3}-\mathrm{Ph}\right)$ ee $=97-99 \%$ (Schiff base ester alkylation)

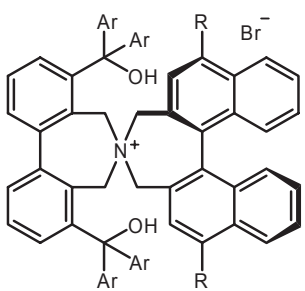

(S)-24a $(\mathrm{Ar}=\mathrm{Ph}, \mathrm{R}=\mathrm{H})$ (S)-24b $(\mathrm{Ar}=\mathrm{R}=3,5$-diphenyl-phenyl) ee up to $96 \%$ (extan (Michael addition of malon

25 : ee up to $83 \%$ (Schiff base ester alkylation)

Fig. 6.

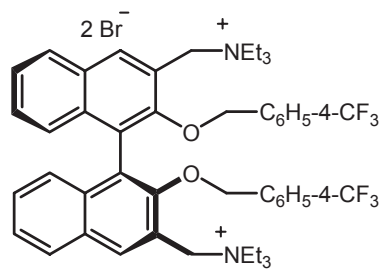

(S)-26: ee up to $75 \%$ (Michael addition on $\alpha, \beta$-unsaturated esters

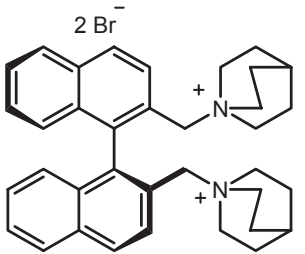

(R)-27: de up to $78 \%$ cis/trans $e_{(\text {cis })}=62 \%$; ee $e_{(\text {trans })}=60 \%$ (Darzens reaction)

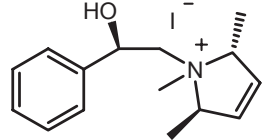

28: ee up to $49 \%$ (Schiff base ester alkylation)
$(\overbrace{\mathrm{Pr}}^{\mathrm{OH}})_{N_{\mathrm{Bn}}^{\mathrm{OTf}}}^{\mathrm{OT}^{-}}$

29: ee up to $58 \%$ (Schiff base ester alkylation)
Fig. 7. on a rigid 6,6'-dimethyl biphenyl skeleton, was also described [50]. For $\mathbf{1 8}$ and 19, bulkier aromatic moieties could be introduced on the biaryl core due to the reduction in steric hindrance around the central nitrogen atom.

Quite a few other cyclic biaryl ammonium cations were reported recently by the groups of Maruoka and Lygo which include (i) configurationally rigid (atropos) [51][52] monobinaphthyl ammonium structures (20 and 21) [53] [54], (ii) atropos monobiphenyl adducts (e.g. 22) [55], (iii) configurationally labile (tropos) biphenyl moieties with either stereogenic exocyclic appendages (e.g. 23) [56] or atropos biaryl moieties (24) [57]. A polycationic variant (25) of catalyst $\mathbf{2 1}$ was also described [58].

Finally, geometrically related yet structurally different dicationic bisammonium catalysts based on a binaphthyl core were also reported recently (26 and 27, Fig. 7) [59][60]. Pyrolinium salt 28 and $C_{3}$-symmetrical ammonium 29 made from enantiopure epoxides precursors were also described. All these moieties displaying moderate to decent levels of selectivity (vide infra) [61][62].

\subsection{Readily Recyclable Ammonium Catalysts}

Further work on polymer-supported phase transfer ammonium catalysts has recently appeared [63]; these catalysts present several advantages over homogeneous catalysts such as simplified work-up for product purification, easy recovery of the catalyst for potential recycling, good stability and reduced toxicity. As before, the novel catalysts are cinchona-based. Several positions for anchoring the polymers were tested (Fig. 8) as well as different spacers (size, length) and polymer supports (Merrifield, PEG or SynPhase lantern).

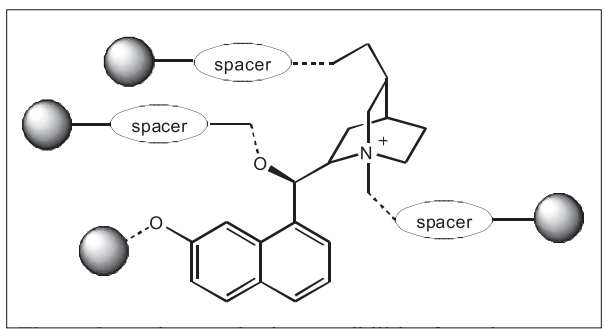

Fig. 8. Various anchoring possibilities for polymer-supported cinchona-based ammonium catalysts

Other recyclable catalysts have been reported in the literature such as fluorousphase compatible $D_{2}$-symmetric ammonium salt 30 (Fig. 9) and dendritic cinchona-based salt 31 (Fréchet-type wedges up to generation three); applications of these catalysts being described later in the article [64][65]. 


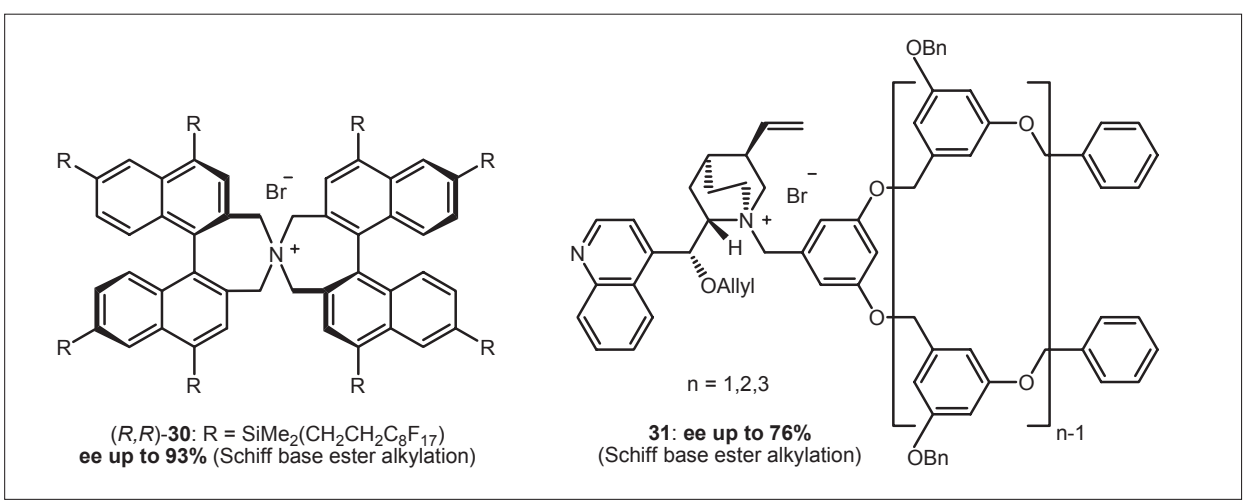

Fig. 9.

After this summary of the variety of the ammonium cations prepared for use in enantioselective PTC, the reactions that were studied will now be described.

\section{Reactions on Schiff Base Glycine 1}

As described, phase transfer catalyzed alkylation of glycine ester derivatives is a powerful method for the preparation of natural and unnatural $\alpha$-amino acids (Scheme 2). As such, most reported studies have used this process as an 'acid test' to measure and compare the efficiency of their enantioselective catalyst system. The most used substrate in this reaction is the glycine Schiff base $t$-butyl ester 1 that usually affords the best reactivity/selectivity profile - although some other substrates have been used (vide infra) [14][66]. Typical conditions are those of reactions performed at $0{ }^{\circ} \mathrm{C}$ with $1 \mathrm{~mol} \%$ of catalyst in a toluene $/ 50 \%$ aqueous $\mathrm{KOH}$ mixture as biphasic solvent medium; completion being reached within a few hours of reaction.

\subsection{Alkylation Reactions with Ammonium Cations Coming from the Chiral Pool}

As mentioned, good levels of enantioselectivity were reached prior to 2003 with ammonium cations of type 2 . In an attempt to provide the most complete view on substituent effects, a general screening of $\mathrm{cin}$ chona-based catalysts with various appendages linked to the nitrogen bridgehead atom and/or the hydroxyl group was performed using a parallel synthesizer and online HPLC monitoring of both rate and enantiomeric excess [67]. Among the 88 catalysts tested, four of them displayed enantiomeric excesses above $90 \%$ with good reproducibility. With such a large data collection, a computational rationalization could then be attempted. Based on twelve structures, comparison of experimental and theoretical enantiomeric excesses was performed and showed a good level of agreement using 3D-QSSR models. This provides a ratio- nalization and a guide to synthetic efforts towards the elaboration of the most efficient catalysts of type 2 [68].

Tartrate derivatives of type $\mathbf{1 6}$ are new to the period 2003-2006. Concerning the alkylation reaction on $\mathbf{1}$, the best catalyst in this series is compound 16a, which provides a high level of enantioselectivity (up to $94 \%$ ee) [42]. As these compounds are advantageous in terms of catalyst accessibility (only five steps amenable to large-scale production using inexpensive reagents and simple operations) and versatility (tunable ketal substituents and aryl groups), a bright future can be envisioned for them.

\subsection{Alkylation Reactions with Pure- ly Synthetic Ammonium Cations}

With purely synthetic ammonium catalysts that often require a synthesis with several steps, care was taken recently to find conditions affording high reactivity and selectivity using truly minimal amounts of catalysts $(<0.1 \mathrm{~mol} \%)$. With compounds of type 3, a screening of various aryl moieties at the 3,3'-positions revealed that the bis $\left(3,4,5-\mathrm{F}_{3}-\mathrm{Ph}\right)$ derivative $\mathbf{3} \mathbf{c}$ is one of the best catalysts for the alkylation of $\mathbf{1}$ since essentially enantiopure natural and unnatural $\alpha$-amino acids can be synthesized. Using anaerobic conditions, it was shown that the overall efficiency of $\mathbf{3} \mathbf{c}$ remains when its loading is decreased to only $0.2 \mathrm{~mol} \%$ [46]. Further improvements could be enacted when the rather lipophilic nature of compounds of type $\mathbf{3}$ was acknowledged. The lipophilicity disfavors the first step of the catalytic process i.e. the interfacial deprotonation of the glycine Schiff base ester 1 by the aqueous base - the cationic ammonium counterion remains in the organic layer rather than moving to the more polar interface or even aqueous layer. Maruoka and coworkers showed that the process can be accelerated using an achiral co-catalyst, 18-crown-6, which helps to extract the inorganic base (usually $\mathrm{KOH}$ ) to the interface or the organic layer. The catalyst and co-catalyst loadings can then be both decreased to $0.05 \mathrm{~mol} \%$ without loss of yield and selec- tivity ( $3 \mathrm{~h}$ reaction time) [69]. Rather than adding a co-reagent, it is also possible to decrease the catalyst loading by increasing the polarity (decreasing the lipophilicity) of the ammonium cation. Using compounds 21 and 22, amounts as low as $0.01 \mathrm{~mol} \%$ could be used and resulted in high yields and enantioselectivity [54][55].

Further studies were performed with compounds of type 3 . One of them was an inquiry on the 'multiplier effect of chiral auxiliaries' using polycationic derivative 25. The length of alkyl chains linking the various ammonium binaphthyl units has a distinct influence on the enantioselectivity (up to $83 \%$ ) and - more surprisingly - also on the absolute configuration of the alkylated product [58].

It was also shown that the presence of an atropos biaryl moiety is not mandatory to achieve high enantioselectivity in the alkylation of $\mathbf{1}$; reactions in the presence of ammonium ions of type $\mathbf{2 3}$ proceeded in some instances with high enantiomeric excess. Compounds of type $\mathbf{2 3}$ combine a tropos biphenyl 7-membered ring and a stereogenic acyclic appendage; Lygo et al. showed that the most efficient catalyst is a combination of a stereogenic 1-(naphthalen-1-yl)ethanamine appendage and two bis(3,5-trifluoromethyl)phenyl moieties at the 3,3' positions of the biphenyl [56]. A computational QSSR experiment was also performed on 40 different catalysts of type 23. Again, good agreement between experimental and theoretical enantiomeric excesses was observed [70].

\subsection{Alkylation Reactions Using Recyclable Ammonium Catalysts or Practical Reaction Conditions}

Studies on the enantioselective alkylation reactions of $\mathbf{1}$ with recyclable ammonium catalysts were further developed in recent years and promising results were obtained on various fronts. For instance, with non-racemic polymer-supported catalysts, enantiomeric excesses as high as $81 \%$ were reported using an organic-layer soluble noncross linked PEG N-bound cinchonidinium salt [71]. An O-bound Merrifield polymer with an $\mathrm{N}$-(9-anthracenylmethyl)cinchon idinium unit displayed very high selectivity (ee up to $96 \%$ ) and recyclability (three times with almost no loss of activity) [63]. Further examples showing similar results in terms of selectivity and reactivity were also reported [72][73].

Fluorous-phase compatible $D_{2}$-symmetric ammonium salt $\mathbf{3 0}$ was also used in the enantioselective alkylation reaction of $\mathbf{1}$. The reactions were performed using standard biphasic conditions (toluene/50\% $\mathrm{KOH}$ aq.) and the catalyst was readily recovered by simple extraction in FC-72 as a fluorous solvent. Enantiomeric excesses 
up to $90 \%$ were obtained and the catalyst could be recycled three times with no loss of activity [64].

Dentritic molecules of type $\mathbf{3 1}$ were also used due to their easy recovery by means of ultra filtration, nanofiltration or size exclusion techniques. Under slightly modified conditions (toluene/ $\mathrm{CHCl}_{3} / 50 \% \mathrm{KOH}$ aq., $-20{ }^{\circ} \mathrm{C}$ ), good yields and decent selectivity levels (up to 76\%) were obtained [65]. Reactions performed under 'tea bag' dialysis membrane conditions required longer reaction times and a loss of selectivity was also noticed after each run (from 64 to $40 \%$ ee after the third run).

Recently, Jew, Park and coworkers showed that practical reaction conditions could be performed by changing the nature of the substrate rather than modifying the catalyst. Polymer-supported glycine Schiff base substrates were used [74]. Anchoring the substrate on Merrifield resins was performed through the imine moiety. The phase transfer alkylation reaction proceeded almost quantitatively with very good enantiomeric excess (up to 99\%) using catalyst $\mathbf{2 b}$ [75].

Finally, Koshima showed that enantioselective phase transfer catalyzed reactions of $\mathbf{1}$ proceeded smoothly on clays and alumina at room temperature to afford alkylated products in high yields and good enantioselectivity [76][77].

\subsection{Conjugate Addition Reactions}

In the above-mentioned reactions, the electrophilic reagents used in combination with substrate $\mathbf{1}$ and the non-racemic ammonium catalysts have been traditional alkyl halides as well as allylic and benzylic moieties. To extend the scope of this chemistry, other electrophiles were used and electron-deficient olefins in particular (Scheme 3, EWG: electron-withdrawing group) [78][79].
For instance, using catalyst 23, Lygo et $a l$. were able to perform the conjugate addition of the enolate of $\mathbf{1}$ to various methyl vinyl ketones; the reaction proceeding with surprisingly modest selectivity (up to $60 \%$ ee). However, a simple modification of the nature of the substrate (a benzhydryl ester instead of a $t$-butyl ester) afforded much higher selectivity (up to 94\%) and, after a simple hydrogenation reaction $\left(\mathrm{H}_{2}, \mathrm{Pd} / \mathrm{C}\right)$, the synthesis of non-racemic 2,5-disubstituted pyrrolidines was readily achieved [80].

Michael addition reactions onto $\alpha, \beta$ unsaturated esters were also realized and ammonium salts $\mathbf{1 6 b}$ and $\mathbf{2 6}$ proved to be quite efficient catalysts for this process as enantiomeric excesses of 82 and $75 \%$ were obtained respectively [42][59]. Another interesting example was reported which concerns the tandem conjugate addition/elimination of activated allylic acetate aimed at synthesizing non-racemic derivatives of glutamic acid (Scheme 4). Enantiomeric excesses up to $97 \%$ were obtained using catalyst $\mathbf{2 b}$ [81].

\subsection{Aldol and Mannich Reactions}

Enantioselective PTC is not limited to the alkylation or conjugate addition reactions of glycine esters of type $\mathbf{1}$ and any reaction proceeding through the formation of a prochiral anionic intermediate upon reaction with a base of the strength of hydroxide $\mathrm{OH}^{-}$is amenable to it. In this context, asymmetric aldol reactions of glycine ester derivatives with aldehydes moieties were tested; these reactions affording a straightforward route to $\beta$-hydroxy- $\alpha$-amino acids which are compounds of great importance in natural product and industrial chemistry (Scheme 5). Using the right combination of aldehydes and catalyst (3e), Maruoka and coworkers were able to obtain high diastereomeric excesses in favor of anti-aldol adducts (up to $92 \%$ ); the major diastereomers being almost enantiopure (ee 98\%) [82].

Similarly, Mannich reactions of glycine ester 1 were performed under enantioselective PTC conditions with imine derivatives as electrophiles. $\alpha, \beta$-Diamino acids were readily afforded (Scheme 6). Two different reports described this chemistry.

In one instance, using imino esters as electrophiles (Scheme 6, $\mathrm{R}^{1}=\mathrm{CO}_{2} \mathrm{Et}, \mathrm{R}^{2}=$ PMP), it was possible to synthesize the nitrogen analogues of diethyl tartrates, which are useful building blocks in modern asymmetric chemistry. Performing the reaction with $2.5 \mathrm{~mol} \%$ of catalyst $\mathbf{3 c}$ under optimized conditions (mesitylene $/ 17 \% \mathrm{NaOH}$ aq., $-20{ }^{\circ} \mathrm{C}, 6 \mathrm{~h}$ ), the syn diastereomer was obtained as the major product with decent diastereomeric (de 64\%) and high enantiomeric excesses (ee 91\%) [83]. In the second case, a solid-liquid PTC protocol was utilized with $10 \mathrm{~mol} \%$ of catalyst $\mathbf{1 6 c}$ and various aromatic imines (Scheme $6, \mathrm{R}^{1}=$ $\left.\mathrm{Ar}, \mathrm{R}^{2}=\mathrm{Boc}\right)$. The syn diastereomer was also obtained predominantly with, again, high levels of selectivity (de 90-99\%, ee $58-82 \%$ ) [44].

\subsection{Structural Variations Around 1}

We have already mentioned that the benzhydryl analogue of ester $\mathbf{1}$ led, in one instance, to a higher level of selectivity than the $t$-butyl ester [80]. Other analogous substrates of 1 were prepared by changing the carboxyl functional group or the nitrogen protecting group. For instance, secondary, tertiary and Weinreb amides were used successfully in combination with catalysts of type 3 (Fig. 10) [84-87]. It was then even possible to perform an iterative process to generate a small peptidic side chain [88]. Modifications of the nitrogen environment were also studied. Very high levels of selectivity were observed using an oxazoline group instead of the diphenylmethanimine group of $\mathbf{1}$ (ee up to $99 \%$ with catalyst 3c) [89].

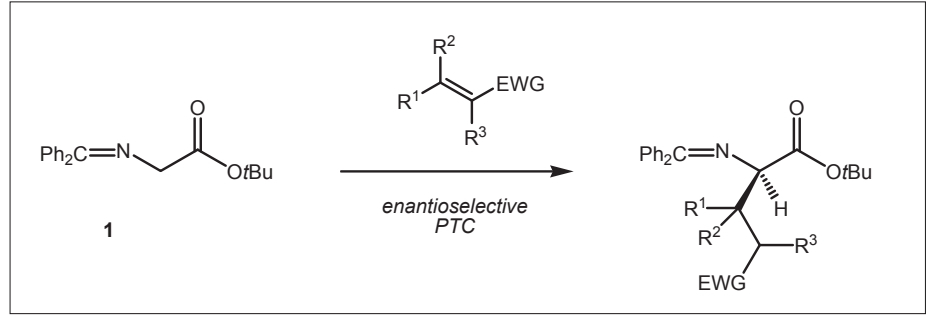

Scheme 3.

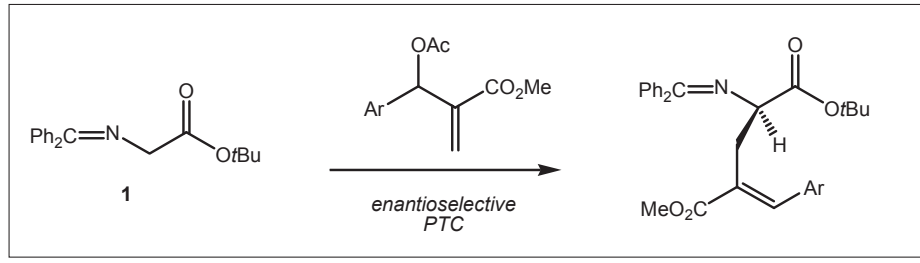

Scheme 4.

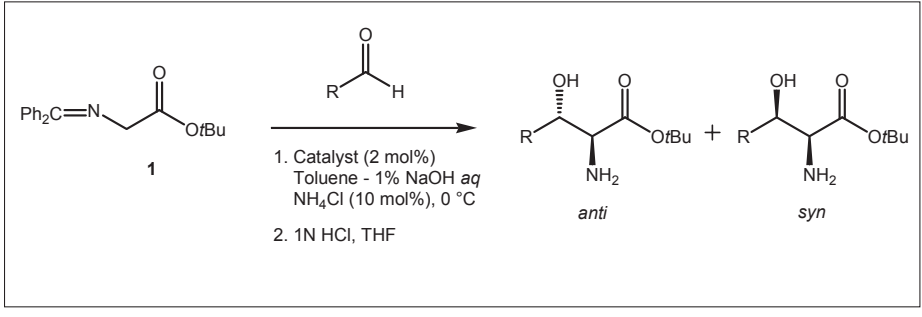

Scheme 5.

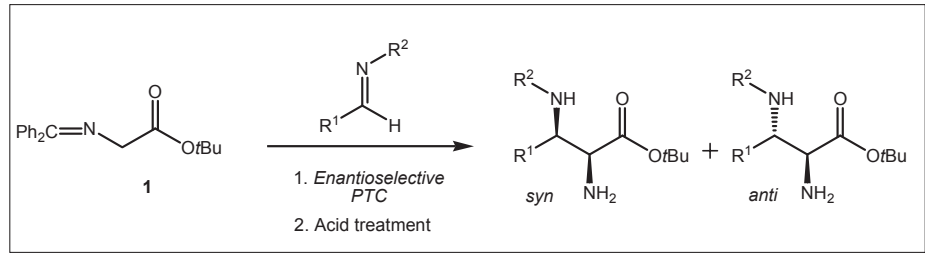

Scheme 6. 


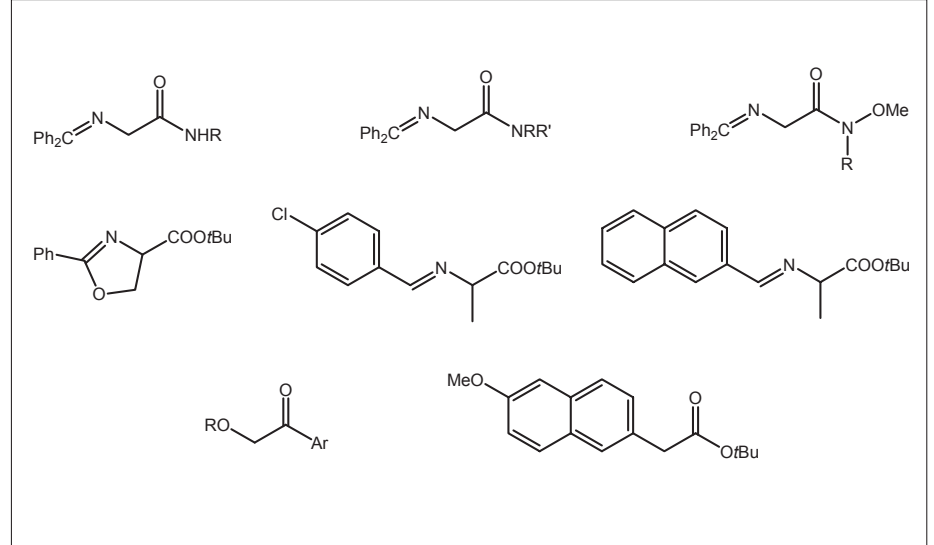

Fig. 10.

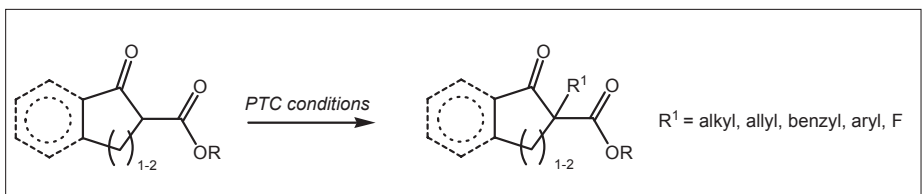

Scheme 7.

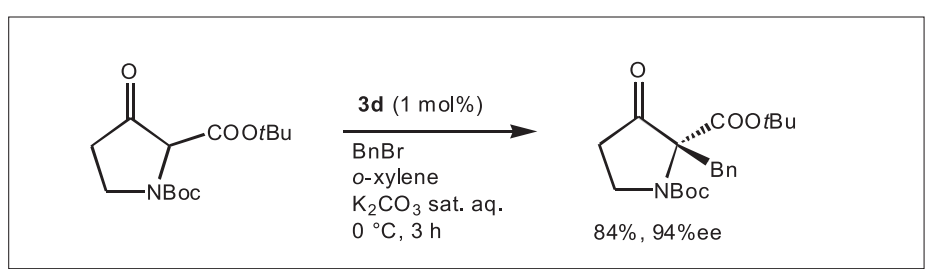

Scheme 8.
To perform dialkylation reactions, the diphenylmethanimine moiety can be replaced by a more reactive (4chlorophenyl)methanimine group [90]. Catalysts 3c, 16, 21 and 24 were then successfully used for the preparation of $\alpha, \alpha^{\prime}$-dialkyl amino acids [42][54][55][91]. By screening various other imine functions, it was shown that the (2-naphthyl)methanimine group is also a good nitrogen protection for dialkylation reactions [92]. Finally, it is also possible to perform alkylation [93][94] and aldol reactions [95] on glycolate adducts and simple ester functions [96].

\section{Other Enantioselective PTC Using Non-racemic Ammonium Salts}

\subsection{Reactions of $\beta$-Keto Esters}

$\beta$-Keto esters are also ready candidates for phase-transfer catalyzed reactions due to the relatively high acidity of the $\alpha$ proton(s) (pKa 14 in DMSO) [97]. Quite a few cyclic variants have been tested in enantioselective reactions; the moieties being aromatic or not, and often 5- or 6-membered rings (Scheme 7).

For instance, using indanone ester derivatives and $1 \mathrm{~mol} \%$ of catalyst 3d, very good yields and enantioselectivity (up to 95\%) were obtained in alkylation reactions. In the same report, it was shown that cyclopentanone and cyclohexanone esters were also capable of performing alkylation reactions and Michael additions onto $\alpha, \beta$-unsaturated aldehydes and ketones with good yields and enantiomeric excesses [98]. Similar results were obtained with various $\beta$-keto esters using catalyst 7a (ee up to 99\%) [99]. Interesting examples of fluorination (catalyst $7 \mathbf{b}$, ee up to $69 \%$ ), and $S_{\mathrm{N}}$ arylation (catalyst of type 2, ee up to $92 \%$ ) of $\beta$-keto ester have also been reported [100][101]. Cyclic $\alpha$-amino- $\beta$-keto esters were also tested in enantioselective PTC to produce azacyclo$\alpha$-amino acids (Scheme 8); catalyst $3 \mathbf{d}$ being highly selective in that process [102].

\subsection{Reactions on Chalcone (and Other Enone) Derivatives \\ 4.2.1. Epoxidation Reactions}

The epoxides of chalcone derivatives are frequently encountered structures in natural product chemistry [103-106]. Since the pioneering work of the Wynberg group who first performed enantioselective phase transfer epoxidation reactions of electrondeficient olefins (Scheme 9) [107], a number of useful variants of this reaction has been elaborated [108][109]. For instance, Lygo et al. (using sodium hypochlorite as oxidant) and Corey et al. (using freshly prepared potassium hypochlorite) were able to perform the reaction with increased level of selectivity using ammonium salts of type 2 as catalysts [110][111].

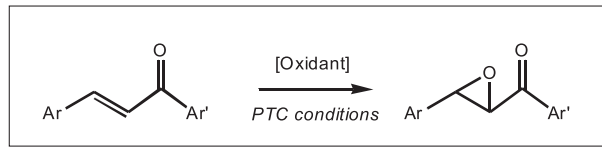

Scheme 9 .

Since then a variety of other oxidants has been used in biphasic or triphasic conditions such as hydrogen peroxide, alkyl hydroperoxide, combinations of urea and $\mathrm{H}_{2} \mathrm{O}_{2}$, sodium perborate or percarbonate, as well as trichloroisocyanuric acid [112][113].

Recently, bifunctional ammonium catalysts 24a and 24b were used to improve further the enantioselectivity of the reactions; these cations being designed to interact simultaneously with both nucleophile and electrophile using electrostatic and $\mathrm{H}$ bonding interactions. A screening of several enones gave systematically excellent yields and enantioselectivity with sodium hypochlorite as the oxidant [57].

In many of these examples, it is necessary to perform the reactions at lower temperature and slower kinetics often results. To recover some of the reactivity, it is possible to add surfactants that increase the surface area between the separated phases by micellar formation [114]. For instance, catalytic amounts of TRITON X-100 or SPAN 200 are sufficient to promote such an effect and, in the presence of a dimeric catalyst of type $\mathbf{1 0}$, rapid quantitative epoxidation of trans-chalcone was observed with quantitative yields and almost complete stereocontrol [34].

\subsubsection{Michael Additions}

As only few reports have appeared on phase-transfer catalyzed enantioselective $\mathrm{C}-\mathrm{C}$ bond forming reactions using chalcones as electrophiles [6][115][116], a recent study tackled the issue using bifunctional catalysts of type $\mathbf{2 4}$; the presence of the alcohol moiety of the ammonium ions being crucial to obtain high levels of selectivity. For instance, using 24b and diethyl malonate as nucleophile, the products of addition onto a variety of chalcones could be isolated with high enantiomeric excesses (from 85 to $94 \%$ ee) [117]. In an independent study, it was shown that cyclopentenones can also be used as electrophiles; addition of malonate esters in presence of catalysts of type 2 occurring with decent selectivity (up to $74 \%$ ) [118][119].

With the aim of synthesizing non-racemic $\gamma$-keto acids, which are important intermediates for the preparation of peptide isosteres, the dimerization of $\alpha, \beta$-enones under enantioselective PTC was recently reported using a Michael - double bond transposition sequence (Scheme 10). With the right combination of enone structure ( $\mathrm{X}$ = electron-donating group, $\mathrm{R}=$ bulky substituent) and catalyst (2c), good yields $(>79 \%)$ and high levels of selectivity (83 to $98 \%$ ee) were obtained [120].

\subsection{Other Reactions}

Enantioselective variants of the azaHenry (or nitro-Mannich) reaction have also been developed under PTC conditions in the presence of non-racemic ammonium salts. Palomo, Bernardi and coworkers have 


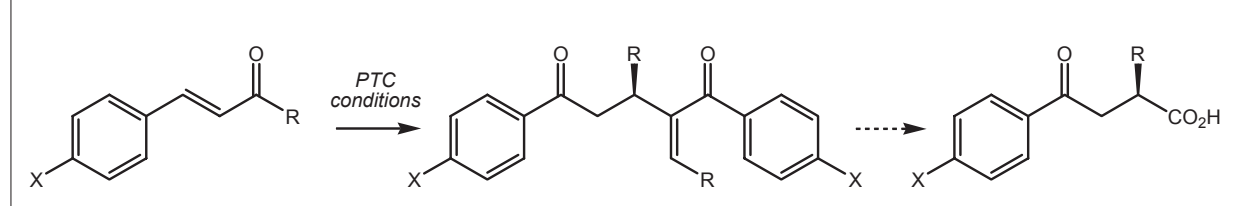

Scheme 10.

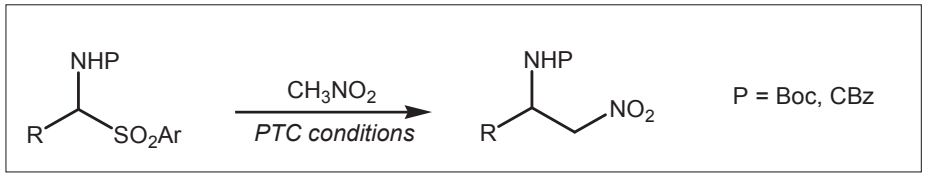

Scheme 11

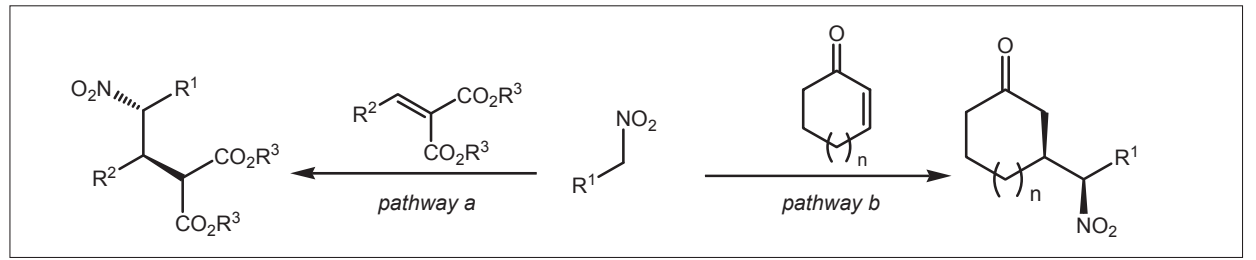

Scheme 12.

(a)

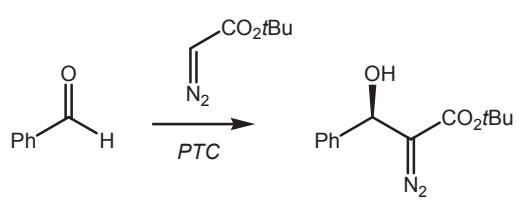

(b)<smiles>[X]CC(=O)Nc1ccccc1</smiles>
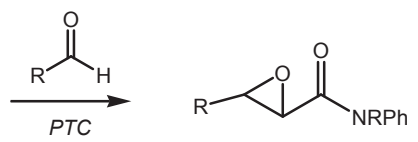

(c)

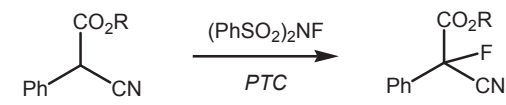

(d)<smiles>[R]C(=O)N(O)[Al]</smiles>
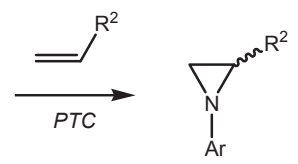

(e)

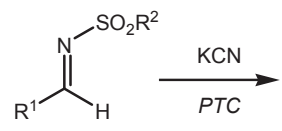

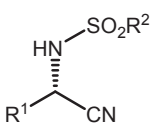

In another report, the fluorination of $\alpha$ cyano esters in the presence of N-fluorobenzenesulfonimide and substoichiometric amounts of $\mathbf{7 b}$ was studied (Scheme 13, reaction (c), ee up to 76\%) [30]. Two unprecedented reactions were also successfully performed under enantioselective PTC that are (i) an aziridination reaction in the presence of catalyst 9 [32], and (ii) a Strecker reaction in the presence of ammonium $\mathbf{2 0}$ [53]; both reactions proceeding with high levels of stereoinduction (Scheme 13, reaction (d), ee up to $95 \%$ and reaction (e), up to $98 \%$ ).

Finally, interesting non-PTC applications of chiral ammonium cations have been developed as the fluoride (or bifluoride) salts of the organic cations catalyze aldol or Michael addition reactions of silyl nitronates or silyl enol ethers [126][127]. It was recently applied to a one-pot stereoselective synthesis of $\gamma$-nitro ketones with three consecutive stereocenters [128].

\section{Acknowledgments}

We thank the University of Geneva, the Swiss National Science Foundation and the State Secretariat for Education and Science for support.

Received: March 15, 2006

[1] M. Makosza, B. Serafin, Rocz. Chem. 1965, 39, 1223.

[2] M. Makosza, A. Jonczyk, Organic Syntheses 1976, 55, 91.

[3] C.M. Starks, R.M. Owens, J. Am. Chem. Soc. 1973, 95, 3613.

[4] C.M. Starks, J. Am. Chem. Soc. 1971, 93, 195.

[5] P.I. Dalko, L. Moisan, Angew. Chem. Int. Ed. 2004, 43, 5138.

[6] R.T. Dere, R.R. Pal, P.S. Patil, M.M. Salunkhe, Tetrahedron Lett. 2003, 44, 5351.

[7] N. Mase, T. Ohno, H. Morimoto, F. Nitta, H. Yoda, K. Takabe, Tetrahedron Lett. 2005, 46, 3213.

[8] L. Li, Z. Zhang, X. Zhu, A. Popa, S. Wang, Synlett. 2005, 1873.

[9] G.D. Yadav, Top. Catal. 2004, 29, 145.

[10] B. Lygo, B.I. Andrews, Acc. Chem. Res. 2004, 37, 518.

[11] K. Maruoka, T. Ooi, Chem. Rev. 2003, 103, 3013.

[12] M.J. O’Donnell, Acc. Chem. Res. 2004, 37, 506 .

[13] U.H. Dolling, P. Davis, E.J.J. Grabowski, J. Am. Chem. Soc. 1984, 106, 446.

[14] M.J. O'Donnell, W.D. Bennett, S. Wu, J. Am. Chem. Soc. 1989, 111, 2353.

[15] M.J. O'Donnell, S. Wu, J.C. Huffman, Tetrahedron 1994, 50, 4507.

[16] B. Lygo, P.G. Wainwright, Tetrahedron Lett. 1997, 38, 8595.

[17] E.J. Corey, F. Xu, M.C. Noe, J. Am. Chem. Soc. 1997, 119, 12414. cyclic $\alpha \beta$ - 12 , pathway a) [123]. With philes, catalyst 3e was more effective in
favor of the syn diastereomers (de up to $96 \%$, ee up to $92 \%$, Scheme 12, pathway [124]. the addition of $t$-butyl diazoacetate to aromatic aldehydes for the one-pot synthesis of $\alpha$-diazo- $\beta$-hydroxyesters; catalysts of type 2 being studied in the context of this reaction (Scheme 13, reaction (a), ee up to $79 \%$ ) [125]. Darzens reaction of $\alpha$-chloro amides in the presence of catalyst $\mathbf{2 7}$ gave glycidic acid derivatives (Scheme 13, reaction (b), ee up to 70\%) [60]. 
[18] B. Lygo, B.I. Andrews, J. Crosby, J.A Peterson, Tetrahedron Lett. 2002, 43, 8015.

[19] S.-S. Jew, B.-S. Jeong, M.-S. Yoo, H. Huh, H.-G. Park, Chem. Commun. 2001, 1244.

[20] R. Chinchilla, P. Mazon, C. Najera, Tetrahedron: Asymmetry 2002, 13, 927.

[21] H.G. Park, B.S. Jeong, M.S. Yoo, M.K. Park, H. Huh, S.-S. Jew, Tetrahedron Lett. 2001, 42, 4645.

[22] R. Chinchilla, P. Mazon, C. Najera, Tetrahedron: Asymmetry 2000, 11, 3277.

[23] B. Thierry, J.-C. Plaquevent, D. Cahard, Tetrahedron: Asymmetry 2001, 12, 983.

[24] B. Thierry, T. Perrard, C. Audouard, J.-C. Plaquevent, D. Cahard, Synthesis 2001, 1742

[25] T. Ooi, M. Kameda, K. Maruoka, J. Am. Chem. Soc. 1999, 121, 6519.

[26] H.U. Blaser, Chem. Rev. 1992, 92, 935.

[27] S. Elango, M. Venugopal, P.S. Suresh, Eni, Tetrahedron 2005, 61, 1443.

[28] S. Kumar, U. Ramachandran, Tetrahedron 2005, 61, 7022.

[29] M.-S. Yoo, B.-S. Jeong, J.-H. Lee, H.-G. Park, S.-S. Jew, Org. Lett. 2005, 7, 1129.

[30] E.J. Park, H.R. Kim, C.U. Joung, D.Y. Kim, Bull. Korean Chem. Soc. 2004, 25, 1451

[31] M.B. Andrus, Z. Ye, J. Zhang, Tetrahedron Lett. 2005, 46, 3839.

[32] E. Murugan, A. Siva, Synthesis 2005, 2022

[33] H.-G. Park, B.-S. Jeong, M.-S. Yoo, J.H. Lee, B.-S. Park, M.G. Kim, S.-S. Jew, Tetrahedron Lett. 2003, 44, 3497.

[34] S.-S. Jew, J.-H. Lee, B.-S. Jeong, M.-S. Yoo, M.-J. Kim, Y.-J. Lee, J. Lee, S.-H Choi, K. Lee, M.S. Lah, H.-G. Park, Angew. Chem. Int. Ed. 2005, 44, 1383.

[35] A. Siva, E. Murugan, J. Mol. Catal. A: Chem. 2005, 241, 111.

[36] A. Siva, E. Murugan, Synthesis 2005, 2927.

[37] S. Kumar, M.E. Sobhia, U. Ramachandran, Tetrahedron: Asymmetry 2005, 16, 2599.

[38] S. Kumar, U. Ramachandran, Tetrahedron 2005, 61, 4141

[39] M.E. Rueffer, L.K. Fort, D.K. MacFarland, Tetrahedron: Asymmetry 2004, 15 3297.

[40] W.E. Kowtoniuk, D.K. MacFarland, G.N. Grover, Tetrahedron Lett. 2005, 46, 5703.

[41] T. Ohshima, V. Gnanadesikan, T. Shibuguchi, Y. Fukuta, T. Nemoto, M. Shibasaki, J. Am. Chem. Soc. 2003, 125, 11206.

[42] T. Ohshima, T. Shibuguchi, Y. Fukuta, M. Shibasaki, Tetrahedron 2004, 60, 7743.

[43] Y. Fukuta, T. Ohshima, V. Gnanadesikan, T. Shibuguchi, T. Nemoto, T. Kisugi, T. Okino, M. Shibasaki, Proc. Natl. Acad. Sci. USA 2004, 101, 5433.

[44] A. Okada, T. Shibuguchi, T. Ohshima, H. Masu, K. Yamaguchi, M. Shibasaki, Angew. Chem. Int. Ed. 2005, 44, 4564.
[45] K. Maruoka, Pure Appl. Chem. 2005, 77, 1285.

[46] T. Ooi, M. Kameda, K. Maruoka, J. Am Chem. Soc. 2003, 125, 5139.

[47] T. Ooi, Y. Uematsu, K. Maruoka, J. Org. Chem. 2003, 68, 4576 .

[48] T. Hashimoto, K. Maruoka, Tetrahedron Lett. 2003, 44, 3313

[49] T. Hashimoto, Y. Tanaka, K. Maruoka, Tetrahedron: Asymmetry 2003, 14, 1599.

[50] T. Ooi, Y. Kubota, K. Maruoka, Synlett. 2003, 1931

[51] K. Mikami, M. Yamanaka, Chem. Rev. 2003, 103, 3369

[52] K. Mikami, K. Aikawa, Y.Yusa, J.J. Jodry, M. Yamanaka, Synlett 2002, 1561.

[53] T. Ooi, Y. Uematsu, K. Maruoka, J. Am. Chem. Soc. 2006, 128, 2548.

[54] M. Kitamura, S. Shirakawa, K. Maruoka Angew. Chem. Int. Ed. 2005, 44, 1549.

[55] Z. Han, Y. Yamaguchi, M. Kitamura, K. Maruoka, Tetrahedron Lett. 2005, 46, 8555.

[56] B. Lygo, B. Allbutt, S.R. James, Tetrahedron Lett. 2003, 44, 5629 .

[57] T. Ooi, D. Ohara, M. Tamura, K. Maruoka, J. Am. Chem. Soc. 2004, 126, 6844.

[58] T. Kano, S. Konishi, S. Shirakawa, K. Maruoka, Tetrahedron: Asymmetry 2004, 15, 1243.

[59] S. Arai, K. Tokumaru, T. Aoyama, Chem. Pharm. Bull. 2004, 52, 646.

[60] S. Arai, K. Tokumaru, T. Aoyama, Tetra hedron Lett. 2004, 45, 1845.

[61] G.N. Grover, W.E. Kowtoniuk, D.K MacFarland, Tetrahedron Lett. 2005, 47, 57.

[62] N. Mase, T. Ohno, N. Hoshikawa, K. Ohishi, H. Morimoto, H. Yoda, K. Takabe, Tetrahedron Lett. 2003, 44, 4073.

[63] B. Thierry, J.-C. Plaquevent, D. Cahard, Molecular Diversity 2005, 9, 277.

[64] S. Shirakawa, Y. Tanaka, K. Maruoka, Org. Lett. 2004, 6, 1429.

[65] G. Guillena, R. Kreiter, R. Van de Coevering, R.J.M. Klein Gebbink, G. Van Koten, P. Mazon, R. Chinchilla, C. Najera, Tetrahedron: Asymmetry 2003, 14, 3705.

[66] B. Lygo, B. Allbutt, Synlett. 2004, 326.

[67] B. Lygo, B.I. Andrews, J.D. Hirst, J.L. Melville, J.A. Peterson, D. Slack, Chimica Oggi 2004, 22, 8.

[68] J.L. Melville, B.I. Andrews, B. Lygo, J.D. Hirst, Chem. Commun. 2004, 1410.

[69] S. Shirakawa, K. Yamamoto, M. Kitamura, T. Ooi, K. Maruoka, Angew. Chem. Int. Ed. 2005, 44, 625

[70] J.L. Melville, K.R.J. Lovelock, C. Wilson, B. Allbutt, E.K. Burke, B. Lygo, J.D. Hirst, J. Chem. Inf. Comput. Model. 2005, 45, 971 .

[71] B. Thierry, J.-C. Plaquevent, D. Cahard, Tetrahedron: Asymmetry 2003, 14, 1671.

[72] R. Chinchilla, P. Mazon, C. Najera, Molecules 2004, 9, 349.

[73] R. Chinchilla, P. Mazon, C. Najera, $A d v$. Synth. Catal. 2004, 346, 1186.
[74] H.-G. Park, M.-J. Kim, M.-K. Park, H.-J. Jung, J. Lee, Y.-J. Lee, B.-S. Jeong, J.H. Lee, M.-S. Yoo, J.-M. Ku, S.-S. Jew, Tetrahedron Lett. 2004, 46, 93.

[75] H.-G. Park, M.-J. Kim, M.-K. Park, H.-J. Jung, J. Lee, S.-H. Choi, Y.-J. Lee, B.-S. Jeong, J.-H. Lee, M.-S. Yoo, J.-M. Ku, S.-S. Jew, J. Org. Chem. 2005, 70, 1904.

[76] H. Yu, H. Koshima, Tetrahedron Lett. 2003, 44, 9209.

[77] H. Yu, S. Takigawa, H. Koshima, Tetrahedron 2004, 60, 8405.

[78] A.H.G. Siebum, R.K.F. Tsang, R. van der Steen, J. Raap, J. Lugtenburg, Eur. J. Org. Chem. 2004, 4391.

[79] R. Chinchilla, P. Mazon, C. Najera, F.J Ortega, M. Yus, ARKIVOC 2005, 222.

[80] B. Lygo, B. Allbutt, E.H.M. Kirton, Tetrahedron Lett. 2005, 46, 4461.

[81] P.V. Ramachandran, S. Madhi, L. BlandBerry, M.V.R. Reddy, M.J. O’Donnell, $J$. Am. Chem. Soc. 2005, 127, 13450.

[82] T. Ooi, M. Kameda, M. Taniguchi, K. Maruoka, J. Am. Chem. Soc. 2004, 126, 9685

[83] T. Ooi, M. Kameda, J. Fujii, K. Maruoka, Org. Lett. 2004, 6, 2397.

[84] T. Ooi, D. Sakai, M. Takeuchi, E. Tayama, K. Maruoka, Angew. Chem. Int. Ed. 2003, 42, 5868

[85] T. Ooi, M. Takeuchi, D. Kato, Y. Uematsu, E. Tayama, D. Sakai, K. Maruoka, $J$. Am. Chem. Soc. 2005, 127, 5073.

[86] S. Kumar, U. Ramachandran, Tetrahedron: Asymmetry 2003, 14, 2539.

[87] S. Kumar, U. Ramachandran, Tetrahedron Lett. 2004, 46, 19.

[88] K. Maruoka, E. Tayama, T. Ooi, Proc. Natl. Acad. Sci. USA 2004, 101, 5824.

[89] S.-S. Jew, Y.-J. Lee, J. Lee, M. J. Kang, B.-S. Jeong, J.-H. Lee, M.-S. Yoo, M.-J. Kim, S.-H. Choi, J.-M. Ku, H.-G. Park, Angew. Chem. Int. Ed. 2004, 43, 2382.

[90] M.J. O'Donnell, B. Leclef, D.B. Rusterholz, L. Ghosez, J.P. Antoine, M. Navarro, Tetrahedron Lett. 1982, 23, 4259.

[91] T. Ooi, Y. Uematsu, K. Maruoka, Tetrahedron Lett. 2004, 45, 1675.

[92] S.-S. Jew, B.-S. Jeong, J.-H. Lee, M.-S. Yoo, Y.-J. Lee, B.-S. Park, M.G. Kim, H.G. Park, J. Org. Chem. 2003, 68, 4514.

[93] M.B. Andrus, E.J. Hicken, J.C. Stephens, D.K. Bedke, J. Org. Chem. 2005, 70, 9470.

[94] M.B. Andrus, E.J. Hicken, J.C. Stephens, Org. Lett. 2004, 6, 2289.

[95] M.B. Andrus, J. Liu, Z. Ye, J.F. Cannon, Org. Lett. 2005, 7, 3861.

[96] S. Kumar, U. Ramachandran, Tetrahedron: Asymmetry 2005, 16, 647.

[97] S. Benettis, R. Romagnolis, C. De Risi, G. Spalluto, V. Zanirato, Chem. Rev. 1995, 95, 1064

[98] T. Ooi, T. Miki, M. Taniguchi, M. Shiraishi, M. Takeuchi, K. Maruoka, Angew. Chem. Int. Ed. 2003, 42, 3796.

[99] E.J. Park, M.H. Kim, D.Y. Kim, J. Org. Chem. 2004, 69, 6897 . 
[100] D.Y. Kim, E.J. Park, Org. Lett. 2002, 4, 545.

[101] M. Bella, S. Kobbelgaard, K.A. Jorgensen, J. Am. Chem. Soc. 2005, 127, 3670 .

[102] T. Ooi, T. Miki, K. Maruoka, Org. Lett. 2005, 7, 191

[103] L. Carde, D.H. Davies, S.M. Roberts, J. Chem. Soc. Perkin Trans. 1 2000, 2455.

[104] B.M. Adger, J.V. Barkley, S. Bergeron, M.W. Cappi, B.E. Flowerdew, M.P. Jackson, R. McCague, T.C. Nugent, S.M. Roberts, J. Chem. Soc. Perkin Trans. 1 1997, 3501.

[105] M.W. Cappi, W.-P. Chen, R.W. Flood, Y.W. Liao, Y.-W.M. Roberts, J. Skidmore, J.A. Smith, N.M. Williamson, Chem. Commun. 1998, 1159.

[106] W.-P. Chen, S.M. Roberts, J. Chem. Soc. Perkin Trans. 1 1999, 103.

[107] R. Helder, J.C. Hummelen, R.W.P.M. Laane, J.S. Wiering, H. Wynberg, Tetrahedron Lett. 1976, 1831.

[108] M.J. Porter, J. Skidmore, Chem. Commun. 2000, 1215.

[109] T. Nemoto, T. Ohshima, M. Shibasaki, J. Synth. Org. Chem. Jpn. 2002, 60, 94.

[110] B. Lygo, P.G. Wainwright, Tetrahedron Lett. 1998, 39, 1599.

[111] E.J. Corey, F.-Y. Zhang, Org. Lett. 1999, 1, 1287.

[112] J. Ye, Y. Wang, R. Liu, G. Zhang, Q. Zhang, J. Chen, X. Liang, Chem. Commun. 2003, 2714.

[113] J. Ye, Y. Wang, J. Chen, X. Liang, $A d v$. Synth. Catal. 2004, 346, 691.

[114] D.J. Shaw, 'Introduction to Colloid and Surface Chemistry', 4th ed., Butterworth-Heinemann, 1992.
[115] A. Loupy, A. Zaparucha, Tetrahedron Lett. 1993, 34, 473.

[116] D.Y. Kim, S.C. Huh, S.M. Kim, Tetrahedron Lett. 2001, 42, 6299.

[117] T. Ooi, D. Ohara, K. Fukumoto, K. Maruoka, Org. Lett. 2005, 7, 3195.

[118] M.I. Donnoli, P. Scafato, M. Nardiello, D. Casarini, E. Giorgio, C. Rosini, Tetrahedron 2004, 60, 4975.

[119] S. Superchi, M. Nardiello, M.I. Donnoli, P. Scafato, R. Menicagli, C. Rosini, C.R. Chimie 2005, 8, 867.

[120] F.-Y. Zhang, E.J. Corey, Org. Lett. 2004 6, 3397.

[121] C. Palomo, M. Oiarbide, A. Laso, R. Lopez, J. Am. Chem. Soc. 2005, 127, 17622.

[122] F. Fini, V. Sgarzani, D. Pettersen, R.P. Herrera, L. Bernardi, A. Ricci, Angew. Chem. Int. Ed. 2005, 44, 7975.

[123] T. Ooi, S. Fujioka, K. Maruoka, J. Am. Chem. Soc. 2004, 126, 11790.

[124] T. Ooi, S. Takada, S. Fujioka, K. Maruoka, Org. Lett. 2005, 7, 5143.

[125] S. Arai, K. Hasegawa, A. Nishida, Tetrahedron Lett. 2004, 45, 1023.

[126] T. Ooi, K. Maruoka, Acc. Chem. Res. 2004, 37, 526.

[127] T. Ooi, K. Morimoto, K. Doda, K. Maruoka, Chem. Lett. 2004, 33, 824.

[128] T. Ooi, K. Doda, S. Takada, K. Maruoka, Tetrahedron Lett. 2006, 47, 145. 